\title{
Determination of the absorption mechanism in photon-induced pre-equilibrium reactions
}

\author{
D. Ryckbosch, L. Van Hoorebeke, R. Van de Vyver, and F. De Smet \\ Laboratorium voor Kernfysica, Rijksuniversiteit Gent, B-9000 Gent, Belgium \\ J-O. Adler, D. Nilsson, B. Schröder, and R. Zorro \\ Department of Physics, University of Lund, Sölvegatan 14, S-223 62 Lund, Sweden
}

(Received 19 March 1990)

\begin{abstract}
Spectra of protons and deuterons emitted in the photodisintegration of ${ }^{27} \mathrm{Al}$ and ${ }^{\text {nat }} \mathrm{Ca}$ have been measured with the tagged photon technique. The experimental data are compared to the results of calculations using the hybrid model for pre-equilibrium reactions. For the first time it is possible to determine the relevant initial doorway configuration, i.e., a $2 \mathrm{p}-1 \mathrm{~h}$ doorway, appropriate for quasideuteron absorption.
\end{abstract}

With the advent of tagged photon facilities with reasonably high photon fluxes, the study of photonuclear reactions in the intermediate energy region $\left(30<E_{\gamma}<140 \mathrm{MeV}\right)$ has received a renewed impetus. In particular, the $(\gamma, p)$ reaction in light nuclei has been studied. Apart from the peaks corresponding to transitions leading to low-lying discrete states in the residual nuclei, a more or less pronounced continuum is observed in the $(\gamma, p)$ spectra. The continuum has, in the case of the lightest target nuclei as, e.g., ${ }^{6} \mathrm{Li}$ and ${ }^{12} \mathrm{C}$, been described in terms of the one-step quasideuteron $(\gamma, n p)$ reaction, where the neutron goes undetected. Due to the stronger final state interaction, however, multistep processes become much more important in heavier nuclei. In such processes the initial doorway state formed in the photon absorption process couples to more complicated states. These states may in turn couple to even more complicated ones, until at last a compound nucleus in thermal equilibrium is formed. By that time, the nucleus has retained little memory of the way it was excited. During the equilibration cascade there is, however, a finite probability for relatively high energetic particles to be emitted. This is the domain of the so-called preequilibrium models, ${ }^{1}$ describing reactions which are intermediate in nature between the two extremes of direct reactions and compound nuclear reactions. The spectra of emitted particles produced in these reactions are harder than the compound evaporation spectra, but much softer than those given by direct reaction models. Of special interest here is, however, the fact that the spectra of these pre-equilibrium-stage particles are still sensitive to details of the initial (photon) absorption mechanism. In this paper we report on an experiment which was aimed at using this sensitivity to study the photon absorption mechanism in two nuclei, ${ }^{27} \mathrm{Al}$ and ${ }^{40} \mathrm{Ca}$.

In the past, a few papers have appeared presenting an analysis of existing photonuclear data in the intermediate energy region in terms of several pre-equilibrium models. ${ }^{2-5}$ These data mostly cover spectra of nucleons ${ }^{6}$ or composite particles ${ }^{4}$ obtained with rather crude bremsstrahlung (difference) methods, or photoneutron multipli- cities $^{7}$ and total photofission probabilities ${ }^{8}$ obtained with quasimonochromatic photons. In general, a good agreement between the theories and the experiment is observed. This was taken by some authors ${ }^{2,3}$ as good evidence for the quasideuteron nature of the photon absorption.

In the intermediate energy region, it is generally assumed that the incoming photon interacts with a (correlated) pair of a neutron and a proton, the so-called quasideuteron. However, the relative importance of this interaction mechanism and of the single-particle absorption mechanism still remains unclear. Absorption of the incoming photon by a single proton will excite a $1 \mathrm{p}-1 \mathrm{~h}$ doorway state. The appropriate doorway state in case of quasideuteron absorption is less obvious. In principle, this should be a $2 \mathrm{p}-2 \mathrm{~h}$ state, whereby the two particles taking part in the absorption process become excited above the Fermi level (but not necessarily immediately emitted to the continuum). However, as was argued in Ref. 3, a more appropriate doorway state could be a 2 p$1 \mathrm{~h}$ state. It is then supposed that the two holes created in the photon absorption remain strongly correlated, acting as a single "effective" degree of freedom.

In Refs. 2 and 4, the experimental data were described using (a version of) the exciton model with an initial $2 \mathrm{p}$ $2 \mathrm{~h}$ doorway state. The authors of Ref. 3, however, found good agreement with the experiment when the hybrid model $^{9}$ is used with an initial $2 \mathrm{p}-1 \mathrm{~h}$ configuration. None of these papers addressed the question of the sensitivity of the calculations to the initial doorway state assumed. This was done for ( $\gamma$, fission) reactions in Ref. 5, and for a much larger set of data covering all photonuclear results obtained with monochromatic photons, in Ref. 10 . It is clear from these studies that, as the reactions available so far all mainly proceed via the compound nuclear stage, the data are very little sensitive to the absorption mechanism. In particular, calculations performed with the hybrid model using $1 \mathrm{p}-1 \mathrm{~h}$ and $2 \mathrm{p}-1 \mathrm{~h}$ initial configurations yield results which are virtually identical and in good agreement with the considered $(\gamma, x n)$ and $(\gamma$, fission) data. Experimental results which are more sensitive to 
the early stages of the reaction are clearly necessary. Such data are provided by the $(\gamma, p)$ and $(\gamma, d)$ spectra measured with monochromatic (tagged) photons reported on here.

Tagged photons were produced at the Lund MAX-Lab tagged photon facility, extensively described elsewhere. ${ }^{11}$ The MAX accelerator system consists of a $100 \mathrm{MeV}$ injector racetrack microtron and a stretcher ring. The $1 \mu \mathrm{s}$ long pulse from the injector is stretched to about $20 \mathrm{~ms}$ which leads, for a repetition rate of $50 \mathrm{~Hz}$, to a $100 \%$ duty cycle. Under normal running conditions, the duty cycle is about $80 \%$ with average currents of up to $50 \mathrm{nA}$ on the $50 \mu \mathrm{m} \mathrm{Al}$ bremsstrahlung radiator. In the present experiment the focal plane of the tagging spectrometer was equipped with 16 individual scintillators. The tagging efficiency $\epsilon_{\text {tagg }}$ was measured with a Pb-glass detector in the photon beam (with suitably reduced intensity). With a photon beam collimated to yield a beam spot of $23 \mathrm{~mm}$ diameter on the photonuclear reaction target, $\epsilon_{\text {tagg }}$ amounts to about $35 \%$. For the $75 \mathrm{MeV}$ incident electrons used in the present experiment, the tagging range extends from 59.3 to $65.2 \mathrm{MeV}$ photon energy.

The photonuclear reaction targets used were a 27.04 $\mathrm{mg} / \mathrm{cm}^{2}$ Al foil and a $45 \mathrm{mg} / \mathrm{cm}^{2}$ (natural) Ca foil with $120 \mu \mathrm{g} / \mathrm{cm}^{2} \mathrm{Al}$ backings. Protons and deuterons emanating from the target were detected in a Si-Ge detector telescope, placed at $90^{\circ}$ with respect to the photon beam (in the laboratory system). The $\Delta E$ detector consisted of a $900 \mathrm{~mm}^{2}, 500 \mu \mathrm{m}$ thick Si detector, while the $E$ counter was a $800 \mathrm{~mm}^{2}, 15 \mathrm{~mm}$ thick hyperpure Ge crystal. The solid angle subtended by this telescope was $\simeq 70 \mathrm{msr}$. Energy calibration of both detectors was performed using a ${ }^{228} \mathrm{Th} \alpha$ source. The reaction targets were mounted in the same vacuum chamber as the detector telescope, which ensured that no material causing energy losses and straggling was present in between the target and the detectors. As such, even relatively low energetic protons and deuterons could be reliably detected in this experiment.

Random coincidences were corrected for in the usual way. The particle spectra obtained in coincidence with the 16 individual focal plane counters were added to yield the netto kinetic energy spectra shown in Figs. 1-3.

In the present work, essentially the same version of the hybrid model code, ALICE/LIVERMORE 82 (Ref. 12), was used as in the study of Ref. 3. In this code, the preequilibrium phase of the reaction is treated using the hybrid model. This amounts to calculating, at each step of increasing complexity of the exciton states, the probability that a particle will be emitted to the continuum, rather than creating a new particle-hole pair. After the complexity of the states has become large enough, thermal equilibrium is assumed and the subsequent decay is described by the Weisskopf-Ewing formalism. Preequilibrium decay was calculated only for protons and neutrons, while the compound nucleus was allowed to decay by evaporation of protons, neutrons, deuterons, and alphas.

Most input parameters (e.g., optical model parameters, level densities, pairing energy treatment, etc.) were taken from the comprehensive evaluation of Ref. 13, where a single parameter set was established for a whole range of nucleon-induced reactions. Throughout the present study, the normal hybrid model was used (as opposed to the Geometry Dependent Hybrid model ${ }^{9}$ ). The internal transition rates $\lambda_{+}$for the creation of a particle-hole pair were calculated on the basis of free nucleon-nucleon cross sections; the nucleon mean free path multiplier $k$ was taken equal to 2, as usual (in the hybrid model). Both types of multiple precompound decay (type I and type II; Refs. 3 and 14) were included in the calculations. Following Blann et al., ${ }^{3,14}$ the hole depth, i.e., the energy which may be taken up by each hole degree of freedom, was limited to the average Fermi energy, i.e., about $30 \mathrm{MeV}$. Consequently, the model and the input parameters used here are known to lead to a good description of nucleonand photon-induced reactions in the energy range of interest. The only parameter that was varied in the present work was the initial doorway configuration, which is

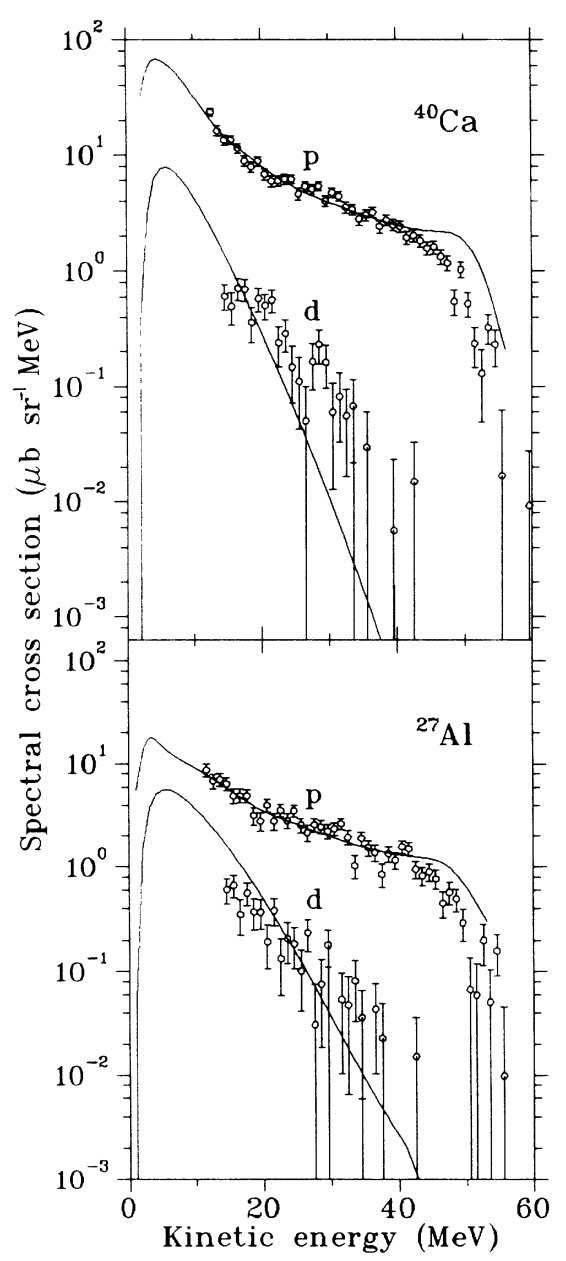

FIG. 1. The $(\gamma, p)$ and $(\gamma, d)$ kinetic energy spectra for ${ }^{\text {nat }} \mathrm{Ca}$ (top) and ${ }^{27} \mathrm{Al}$ (bottom). The tagged photon energy range is from 59.3 to $65.2 \mathrm{MeV}$. The full lines are the results of a calculation with the hybrid pre-equilibrium model with an initial 1p$1 \mathrm{~h}$ configuration. For the deuterons, only equilibrium decay was assumed in the calculation. 
dependent on the nature of the absorption mechanism.

Experimentally, differential spectral cross sections at $90^{\circ}$ have been measured, while the present version of the hybrid model allows the calculation of total spectral cross sections only. However, this should have only a minor influence on the shape of the spectra. The forward peaked character of the angular distributions, typical for the direct reaction channels, is very rapidly damped out under the influence of the final state interactions. Moreover, the angular distributions in photonuclear reactions are in general less pronounced than those typical of nucleon-induced reactions. In the case of protons and for low mass target nuclei, some data are available which cover a relatively large range in particle energy, e.g., Ref. 15. From these data it appears that the continuum part of the particle spectrum is almost isotropic. The same is true for the case of deuterons. ${ }^{4}$ As such, it is clear that a comparison of the shape of the calculated and the experimental spectra is meaningful.

In Figs. $1-3$, the experimental $(\gamma, p)$ and $(\gamma, d)$ spectra for ${ }^{27} \mathrm{Al}$ and $\mathrm{Ca}$ are compared to the model calculations for the three considered different initial configurations. These represent the two main modes of absorption sug- gested for photons, i.e., single-particle absorption (1p-1h, Fig. 1) and quasideuteron absorption (2p-2h, Fig. 2 and $2 \mathrm{p}-1 \mathrm{~h}$, Fig. 3). When comparing the theoretical spectra to those determined experimentally, one should keep in mind the following considerations. In addition to the equilibrium and pre-equilibrium processes described in the model calculation, there are also direct reactions contributing to the $(\gamma, p)$ spectra. Such reactions lead to simple, low-lying states in the residual $A-1$ nucleus. Consequently, one must expect the calculated proton spectra to reproduce the experimental data over the whole energy region, except at the highest energies, say above $45 \mathrm{MeV}$, where theory should lie below the data. With regard to the deuteron spectra, one should expect a reasonable agreement between theory and experiment only at the lowest particle energies, where the compound decay dominates over the pre-equilibrium decay (which was not included in the calculations).

The theoretical curves were normalized to the experimental spectra by minimizing the usual $\chi^{2}$ for proton energies between 15 and $40 \mathrm{MeV}$. The reduced $\chi^{2}$ for the different initial configurations are listed in Table I. From this table and from Figs. 1-3, it is obvious that the best

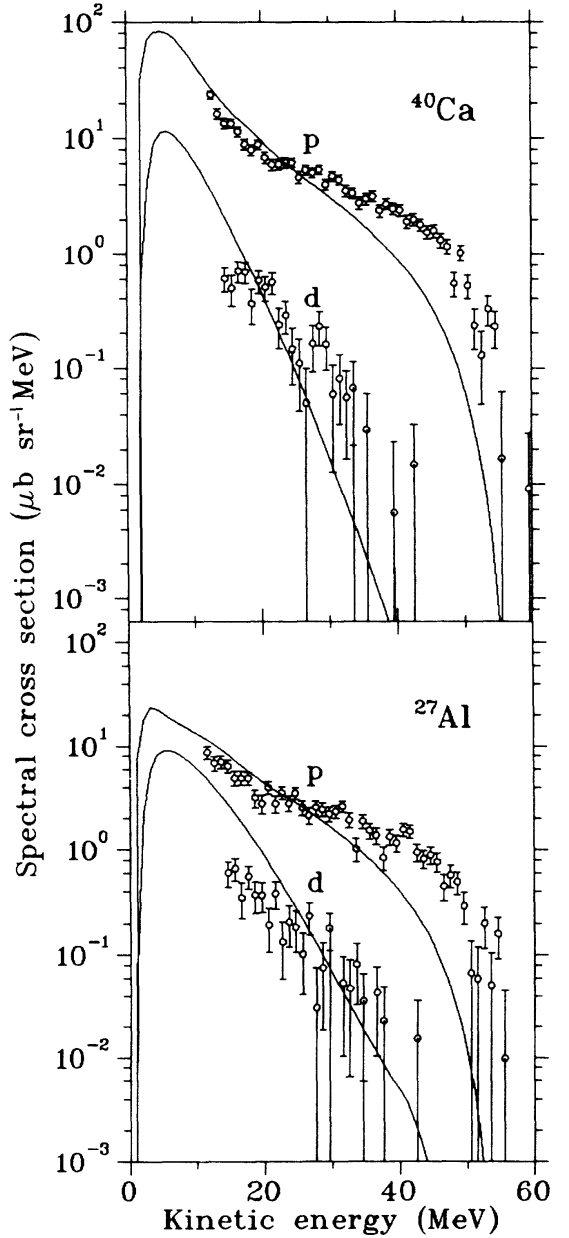

FIG. 2. Same as Fig. 1, with a $2 \mathrm{p}-2 \mathrm{~h}$ initial configuration.

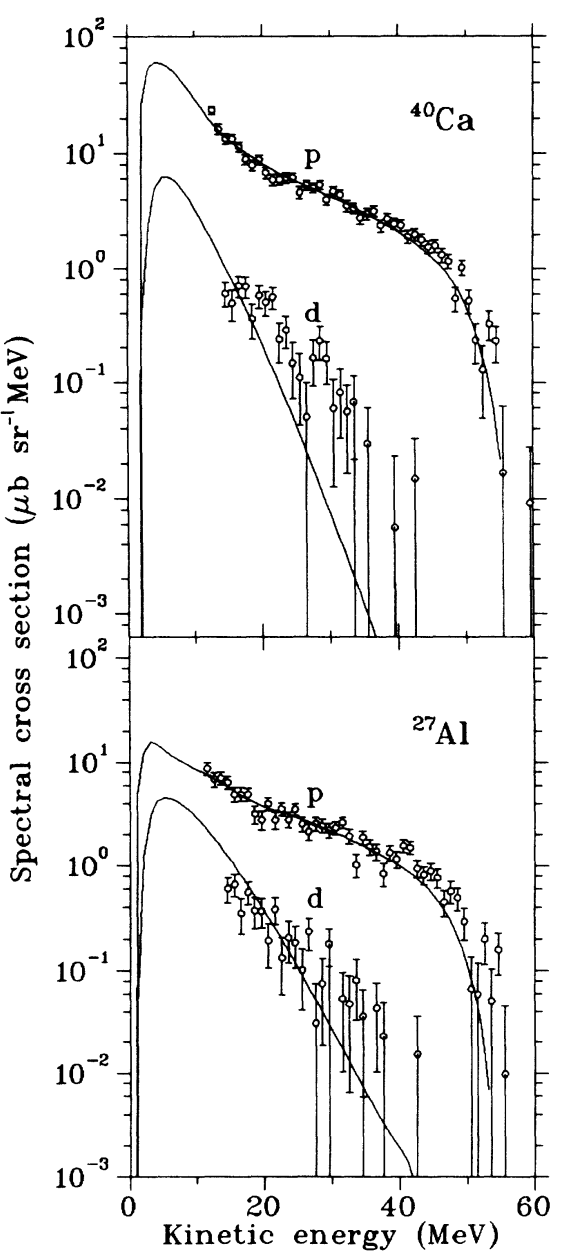

FIG. 3. Same as Fig. 1, with a 2p-1h initial configuration. 
TABLE I. Value of reduced $\chi^{2}$ for the comparison of theoretical and experimental $(\gamma, p)$ spectra between 15 and 40 $\mathrm{MeV}$.

\begin{tabular}{lcc}
\hline \hline Target & ${ }^{27} \mathrm{Al}$ & ${ }^{\text {nat }} \mathrm{Ca}$ \\
\hline $1 \mathrm{p}-1 \mathrm{~h}$ & 1.50 & 1.39 \\
$2 \mathrm{p}-2 \mathrm{~h}$ & 5.19 & 11.4 \\
$2 \mathrm{p}-1 \mathrm{~h}$ & 1.24 & 1.17 \\
\hline \hline
\end{tabular}

description of the experimental spectra is found when a $2 \mathrm{p}-1 \mathrm{~h}$ initial configuration is used. The $2 \mathrm{p}-2 \mathrm{~h}$ initial configuration yields results which are not in agreement with the experimental data. The $1 \mathrm{p}-1 \mathrm{~h}$ initial state gives a fit to the results between 15 and $40 \mathrm{MeV}$ which is only slightly worse than the fit given by the $2 \mathrm{p}-1 \mathrm{~h}$ doorway state. However, it is seen that such a $1 \mathrm{p}-1 \mathrm{~h}$ doorway leads to a drastic overestimation of the $(\gamma, p)$ data at the highest energies. Although the present statistical calculation cannot be expected to describe the data in this energy region, where a direct reaction mechanism certainly also contributes, it should not predict a spectral cross section which is some factor of two higher than what is experimentally found. In contrast, the spectrum calculated with the $2 \mathrm{p}-1 \mathrm{~h}$ initial configuration lies just below the data at the highest energies, precisely what should be expected within the present model. The present $(\gamma, p)$ results thus indicate that a $2 \mathrm{p}-1 \mathrm{~h}$ initial configuration is the most appropriate configuration for a description of preequilibrium photonuclear reactions with the hybrid model.

Another feature of the comparison between theory and experiment is that none of the used initial configurations yields photodeuteron spectra in good agreement with the experiment. However, it is clear that the $2 \mathrm{p}-1 \mathrm{~h}$ initial doorway leads to a reasonable description of the lowest energy part of the deuteron spectra. The other initial configurations overestimate the spectral cross section in this region. At the higher particle energies, the calculations always lie below the experimental data. This is certainly due to the neglect of pre-equilibrium deuteron emission in the calculations. Indeed, one observes that the slopes of the experimental proton and deuteron spectra are rather similar, indicating a common emission mechanism. The main point is, however, that the present
TABLE II. Total photon absorption cross section (in mb) used in the calculations. The photon energy is about $60 \mathrm{MeV}$.

\begin{tabular}{ccc}
\hline \hline Target & $\mathrm{Al}$ & $\mathrm{Ca}$ \\
\hline $1 \mathrm{p}-1 \mathrm{~h}$ & 3.59 & 6.66 \\
$2 \mathrm{p}-2 \mathrm{~h}$ & 4.57 & 7.54 \\
$2 \mathrm{p}-1 \mathrm{~h}$ & 3.15 & 5.77 \\
Experiment $^{\mathrm{a}}$ & $3.6 \pm 0.8$ & $5.4 \pm 1.2$ \\
\hline \hline
\end{tabular}

${ }^{\text {a }}$ Reference 16.

calculation correctly describes the large ratio between $(\gamma, p)$ and $(\gamma, d)$ spectral cross sections. This lends support to the reaction model used here. Obviously, the $(\gamma, d)$ data alone are by far not sensitive enough to the initial absorption process to allow a determination of the initial configuration.

Assuming that the emission of particles was isotropic, the normalization of the calculated spectra to the experimental ones can be used to obtain at least an estimate of the total photon absorption cross section at the present photon energy of about $60 \mathrm{MeV}$. These are given in Table II. The values quoted there are close to what is expected from absorption measurements. ${ }^{16}$

In conclusion, we have presented new photoproton and photodeuteron spectra for ${ }^{27} \mathrm{Al}$ and ${ }^{\text {nat }} \mathrm{Ca}$ obtained with tagged photons, which have been compared to the results of theoretical calculations using the hybrid model. It was shown that for both the $(\gamma, p)$ and the (compound part of the) $(\gamma, d)$ spectra, good agreement was found between theory and experiment when an initial $2 \mathrm{p}-1 \mathrm{~h}$ configuration is used. Other initial configurations, reflecting a different photon absorption mechanism, lead to results in disagreement with the experiment. As such, these data again affirm the dominance (at $60 \mathrm{MeV}$ ) of a mechanism like the quasideuteron model, where two nucleons are effectively involved in the absorption of an incoming photon.

We acknowledge the financial support given by the Interuniversity Institute of Nuclear Sciences (IIKW) and the National Fund for Scientific Research (NFWO), Brussels, Belgium. The work of the Lund collaborators has been supported by the Swedish Natural Science Research Council. We thank the members of the MAX accelerator staff for providing the electron beam.
${ }^{1}$ H. Gruppelaar, P. Nagel, and P. E. Hodgson, Riv. Nuovo Cimento 9, 1 (1986).

${ }^{2}$ J. R. Wu and C. C. Chang, Phys. Rev. C 16, 1812 (1977).

${ }^{3}$ M. Blann, B. Berman, and T. T. Komoto, Phys. Rev. C 28, 2286 (1983).

${ }^{4}$ A. G. Flowers, P. J. Thorley, I. Anthony, D. Branford, J. C. McGeorge, M. R. Sene, A. C. Shotter, C. H. Zimmerman, and R. O. Owens, Nucl. Phys. A429, 61 (1984).

${ }^{5}$ D. Ryckbosch, P. Carlos, and A. Lepretre, Z. Phys. A 329, 451 (1988).

${ }^{6}$ N. N. Kaushal, E. J. Winhold, P. F. Yergin, H. A. Medicus, and R. H. Augustson, Phys. Rev. 175, 1330 (1968).

${ }^{7}$ A. Leprêtre, H. Beil, R. Bergère, P. Carlos, J. Fagot, A. de
Miniac, and A. Veyssière, Nucl. Phys. A367, 237 (1981).

${ }^{8}$ A. Leprêtre et al., Nucl. Phys. A472, 533 (1987).

${ }^{9}$ M. Blann, Phys. Rev. Lett. 27, 337 (1971); 27, 700(E) (1971); 27, 1550 (E) (1971)

${ }^{10}$ D. Ryckbosch, P. Carlos, and A. Leprêtre, Commissariat à l'Energie Atomique Note CEA-N-2582(E), 1988.

${ }^{11} \mathrm{~J}-\mathrm{O}$. Adler et al., Nucl. Instrum. Methods (in press).

${ }^{12} \mathrm{M}$. Blann and J. Bisplinghoff, University of California Report UCID-19614, 1982 (unpublished).

${ }^{13}$ M. Blann and H. K. Vonach, Phys. Rev. C 28, 1475 (1983).

${ }^{14}$ M. Blann, Phys. Rev. C 28, 1648 (1983).

15J. C. McGeorge et al., Phys. Lett. B 179, 212 (1986).

${ }^{16}$ J. Ahrens, Nucl. Phys. A446, 229c (1985). 